\section{Kliniksterben in Davos}

Die Kosteneinsparungen im deutschen Gesundheitswesen zeigen nun auch Wirkung im Ausland. Zwei von drei deutschen Kliniken in Davos, die Höhenklinik Valbella Davos und die Alexanderhausklinik, mussten bereits schließen, die Schweizerische Thurgau-Schaffhausische Höhenklinik wird im April 2005 ihren Betrieb einstellen. Übrig bleiben in Davos somit nur noch die deutsche Hochgebirgsklinik Davos mit Allergieklinik Davos und dem Niederländischen Asthmazentrum Davos (NAD), die Zürcher Höhenklinik Clavadel sowie die Alpine Kinderklinik Davos. Letztere befindet sich allerdings auch in finanziellen Schwierigkeiten und ist auf öffentliche Unterstützung angewiesen. Von den Änderungen im deutschen Gesundheitswesen sind ausschließlich die deutschen Kliniken betroffen. Die Schwierigkeiten der Schweizer Kliniken sind durch nationale Besonderheiten begründet - insbesondere die Streichung öffentlicher Zuschüsse sowie auch versäumte Investitionen für Modernisierungen.

Die als einzige deutsche Einrichtung übrig gebliebene Hochgebirgsklinik Davos profitiert von der aktuellen Situation und nimmt Personal und Patienten von den zu schließenden Kliniken auf. Für den ärztlichen Direktor der Klinik, Priv.-Doz. Dr. Günter Menz, kommt die aktuelle Situation nicht überraschend: „Die Stiftung Deutsche Hochgebirgsklinik Davos verfolgt seit Jahren die Strategie einer Konzentration und hat sich entsprechend positioniert. Den jetzt einsetzenden Prozess haben wir erwartet, denn er war überfällig, das Bettenangebot in Davos für deutsche Patienten schlicht überdimensioniert." Die Klinik kooperiert als Teil des 2003 gegründeten Europäischen Asthma- und Allergie-Zentrums Davos (EACD) auch mit dem Niederländischen Asthmazentrum Davos und dem Schweizer Institut für Allergie- und Asthma-Forschung (SIAF).

\section{Ehrenmitgliedschaft}

Prof. Dr. Dr. Johannes Ring, Direktor der Klinik und Poliklinik für Dermatologie und Allergologie am Biederstein, ist Ende des letzten Jahres zum Ehrenmitglied der rumänischen Gesellschaft für Dermatologie ernannt worden.
Schweiz" auf. Im Rahmen desselben wurden während 20 Jahren umfassende Daten von über 50.000 konsekutiven Patienteneintritten gesammelt und in einem eigens hierfür entwickelten EDVSystem gespeichert. Aus diesem umfangreichen Datenmaterial sind bis heute über 70 Publikationen, teils epidemiologischer Natur, teils über bisher unbekannte oder wenig bekannte Arzneimittelnebenwirkungen in internationalen Zeitschriften erfolgt.

Zudem war Rolf Hoigné seit 1980 Mitherausgeber von „Meyler's Side Effects of Drugs", dem Standardwerk für Arzneimittelnebenwirkungen. Als besondere Ehrungen seien erwähnt: die Präsidentschaft der Schweizerischen Gesellschaft für Allergologie und Immunologie
1964-1966, der Kurt-Adam-Preis der Deutschen Kongressgesellschaft für ärztliche Fortbildung 1974 sowie der Allergie-Staffelpreis 1993 des Forschungsinstitutes Bad Lippspringe. Schliesslich war Rolf Hoigné seit den 50er Jahren Mitglied des Collegium Internationale Allergologicum.

\section{Habemus in memoriam!}

Wir behalten mit Prof. Dr. Rolf Hoigné einen engagierten Arzt und klinischen Lehrer in innerer Medizin, Allergologie und klinischer Immunologie, aber auch einen unermüdlichen und sorgfältigen klinischen Forscher in bleibender Erinnerung

Prof. Dr. Ulrich R. Müller

\title{
Herbert-Herxheimer-Förderpreis 2005
}

Der Herbert-HerxheimerFörderpreis, gestiftet von der DPC Biermann GmbH, Bad Nauheim, in Zusammenarbeit mit der Deutschen Gesellschaft für Allergologie und klinische Immunologie, wird jährlich für herausragende Originalarbeiten verliehen, in diesem Jahr im Rahmen des WorldAllergy Congress, der vom 26. Juni bis 1. Juli 2005 in München stattfindet.

Der Preis ist ausschließlich zur Auszeichnung besonderer wissenschaftlicher Arbeiten auf dem Gebiet der Allergologie und klinischen Immunologie bestimmt und dient der Förderung jüngerer Wissenschaftler. Zurzeit

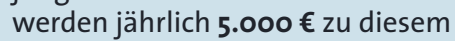
Zweck zur Verfügung gestellt. Der Preis ist für Wissenschaftler aus dem deutschen Sprachraum vorgesehen, die sich noch nicht in Lebensstellung befinden und die das 40. Lebensjahr zum Zeitpunkt der Einreichung der Arbeit noch nicht vollendet haben. Ausgeschlossen sind Angestellte von Industriefirmen. Für eine Prämierung kommen Arbeiten in Frage, die sich mit diagnostischen und therapeutischen Fragestellungen oder der Grundlagenforschung auf den Gebieten der Allergologie und klinischen Immunologie sowie verwandter Gebiete befassen.

Die Bewerber reichen bis zum 30. April 2005 Originalarbeiten, die bis zu diesem Zeitpunkt publiziert oder zur Publikation angenommen sind, in deutscher oder englischer Sprache ein. Die Arbeiten sollen in fünffacher Ausfertigung vorgelegt werden. Eine Verblindung ist nicht erforderlich. Der Erstautor hat handschriftlich zu versichern, dass er die Arbeit selbstständig erstellt hat. Die Jury kann bei mehr als einer eingereichten preiswürdigen Arbeit den Preis teilen. Je Arbeitsgruppe kann nur eine Arbeit eingereicht werden.

\footnotetext{
Deutsche Gesellschaft für Allergologie und klinische Immunologie (DGAI) Frau Erika Ratzinger Postfach 7004 64, 81304 München Tel.: (o 89) 54662968 Fax: (o 89) 583824 E-Mail: ratzingererika@t-online.de
} 